\title{
The family of c-bisections auctions: efficiency and running time
}

Citation for published version (APA):

Vermeulen, A. J., Grigorieva, E., Müller, R. J., \& Herings, P. J. J. (2006). The family of c-bisections auctions: efficiency and running time. METEOR, Maastricht University School of Business and Economics. METEOR Research Memorandum No. 019 https://doi.org/10.26481/umamet.2006019

Document status and date:

Published: 01/01/2006

DOI:

10.26481/umamet.2006019

Document Version:

Publisher's PDF, also known as Version of record

\section{Please check the document version of this publication:}

- A submitted manuscript is the version of the article upon submission and before peer-review. There can be important differences between the submitted version and the official published version of record.

People interested in the research are advised to contact the author for the final version of the publication, or visit the DOI to the publisher's website.

- The final author version and the galley proof are versions of the publication after peer review.

- The final published version features the final layout of the paper including the volume, issue and page numbers.

Link to publication

\footnotetext{
General rights rights.

- You may freely distribute the URL identifying the publication in the public portal. please follow below link for the End User Agreement:

www.umlib.nl/taverne-license

Take down policy

If you believe that this document breaches copyright please contact us at:

repository@maastrichtuniversity.nl

providing details and we will investigate your claim.
}

Copyright and moral rights for the publications made accessible in the public portal are retained by the authors and/or other copyright owners and it is a condition of accessing publications that users recognise and abide by the legal requirements associated with these

- Users may download and print one copy of any publication from the public portal for the purpose of private study or research.

- You may not further distribute the material or use it for any profit-making activity or commercial gain

If the publication is distributed under the terms of Article $25 \mathrm{fa}$ of the Dutch Copyright Act, indicated by the "Taverne" license above, 
Elena Grigorieva, P. Jean-Jacques Herings, Rudolf Müller, Dries Vermeulen

The family of c-bisection auctions: efficiency and running time

$\mathrm{RM} / 06 / 019$

JEL code: C72, D44

\section{METE@R}

Maastricht research school of Economics of TEchnology and ORganizations

Universiteit Maastricht

Faculty of Economics and Business Administration P.O. Box 616

NL - 6200 MD Maastricht

phone : ++31433883830

fax : $\quad++31433884873$ 



\section{The family of $c$-bisection auctions: efficiency and running time}

Elena Grigorieva, ${ }^{1}$ P.Jean-Jacques Herings, ${ }^{2}$ Rudolf Müller, ${ }^{3}$ and Dries Vermeulen ${ }^{4}$

Summary. In this paper we analyze the performance of a recently proposed sequential auction, called the $c$-bisection auction, that can be used for a sale of a single indivisible object. We discuss the running time and the efficiency in the ex-post equilibrium of the auction. We show that by changing the parameter $c$ of the auction we can trade off efficiency against running time. Moreover, we show that the auction that gives the desired level of efficiency in expectation takes the same number of rounds for any number of players.

Keywords and Phrases: query auction, efficiency, running time

JEL Classification Numbers: C72, D44.

\footnotetext{
${ }^{1}$ e.grigorieva@ke.unimaas.nl. Department of Quantitative Economics, Maastricht University, P.O. Box 616, 6200 MD Maastricht, The Netherlands. The author acknowledges support by the Dutch Science Foundation NWO through grant 401-01-101.

${ }^{2}$ p.herings@algec.unimaas.nl. Department of Economics, Maastricht University, P.O. Box 616, 6200 MD Maastricht, The Netherlands. The author acknowledges support by the Dutch Science Foundation NWO through a VICI-grant.

${ }^{3}$ r.muller@ke.unimaas.nl. Department of Quantitative Economics, Maastricht University, P.O. Box 616, 6200 MD Maastricht, The Netherlands. The author acknowledges support by European Commission through funds for the International Institute of Infonomics.

${ }^{4}$ d.vermeulen@ke.unimaas.nl. Department of Quantitative Economics, Maastricht University, P.O. Box 616, 6200 MD Maastricht, The Netherlands.
} 


\section{Introduction}

A central issue in auction design is to set the rules of the auction so that some economic goals are achieved despite the fact that agents act based on self-interest. The economic goals are often expressed as functions of agents' preferences. Therefore auction design needs to elicit information on agent's preferences.

However requiring elicitation of full and exact preference information is undesirable for several reasons. First, agents may prefer not to reveal information on their valuations for reasons of privacy or long-term competitiveness [13]. Second, determining one's valuation with a precision up to the last digit can be computationally demanding $[9,12,14]$. Finally, the full revelation of agents' preferences may require a prohibitive amount of communication $[5,11]$.

Such considerations lead to an interest in auctions where agents need not reveal their information entirely but only partially. It has been recognized that multi-round mechanisms can reduce revelation and associated with it computation and communication, compared to single-round mechanisms advocated by the revelation principle $[1,3]$. One class of multi-round mechanisms are query auctions. In a query auction the auctioneer sequentially queries the agents about specific aspects of their preferences. As an answer to the query an agent can chose one of a finite set of actions. Through incremental querying, the auctioneer gradually collects the information on agents' valuations. By using a query strategy in which previously revealed information guides the selection of subsequent queries, elicitation is focused on pertinent information. Incremental querying has been applied in different settings $([7,2])$ and it has been shown that only a small fraction of agents' valuation information needs to be revealed before the (approximately) optimal allocation can be determined $[5,8]$.

When evaluating the effectiveness of elicitation we may generally care about the running time expressed in the number of queries required to determine an optimal (according to the specific objective of the designer) allocation [15]. Since information about agents' valuation becomes more refined with each query, a higher number of queries leads to a better allocation. This has prompted researchers to examine the trade-off between the running time of query auctions and the level of allocation optimality. For example, in [4] the issue is considered for English auctions when restricting queries to discrete levels. The authors analyze how the choice of query levels in the English auction affects the expected revenue and the expected duration (measured in terms of the number of levels that the query price has been raised through).

The motivation for the topic of research in this paper is as follows. In [6] we study the uses and limitations of query auctions regarding the objective of economic efficiency maximization. In particular, we prove 
that in a setting with valuations distributed according to a continuous density function any ex-post equilibrium in an ex-post individual rational query auction that ends with positive probability after a finite number of queries, can not be fully efficient. This result implies that in the setting of continuous valuations full efficiency can only be achieved at the expense of an infinite running time of a query auction for almost all realizations of valuations. So the question that arises is: what price (in terms of running time) has to be paid for getting a desired level of approximate efficiency.

Thus, in this paper we are concerned with the issue of the trade-off between running time and allocation efficiency in the recently proposed $c$-bisection auction [6]. The proposed auction is a query mechanism in which players submit their bids in sequence with full knowledge of all previous bids of other players. The auction is characterized by a parameter $c$ which, together with the distribution from which valuations of players are drawn, determines a sequence of query prices. In [6] equilibrium properties of this auction are analyzed. It is shown that the auction has an ex-post equilibrium, called the bluff equilibrium, and under this equilibrium the auction ends in finite time, regardless of the realization of players' valuation. Due to the result mentioned above we know that inefficiency of the bluff equilibrium for some realizations of valuations is inevitable.

In this paper we discuss in detail the performance of the $c$-bisection auction under the bluff equilibrium. In particular, we study how the choice of parameter $c$ and the number of participating players affect the running time of the auction and its (in)efficiency.

First, we investigate the running time of the auction according to two measures, namely the expected number of rounds and the expected number of queries performed in the auction. ${ }^{5}$ For both measures we derive first a recursive formula and give then an upper bound for the function defined by this formula. We prove that for a fixed $c$ the expected number of rounds is bounded by a function that is logarithmic in the number of players while the expected number of queries is bounded by a function that is linear in the number of players.

Second, we analyze the level of inefficiency of the auction. As measures of inefficiency we employ the probability of inefficient allocation and the expected loss of welfare. For the probability of inefficient allocation we derive a recursive formula and prove that it's not more than $c$ for any number of players. We show that when valuations are uniformly drawn from $[0,1)$ the expected loss of welfare is bounded from above by $c^{2}$ for any number of players. It means that by choosing the appropriate $c$, the minimum level of efficiency can be determined by the auctioneer before it is known how many players will participate

\footnotetext{
${ }^{5}$ As a query we consider each separate question of the auctioneer to an active player. As a round we consider a sequence of queries in which each active player is asked to act exactly once.
} 
in the auction. We also give a (more) precise estimate of the expected loss of welfare by using computer simulation.

Furthermore we show that for a fixed number of players there is a trade-off between efficiency and running time: for the increasing efficiency of the auction we have to pay by an increasing number of rounds and an increasing number of queries. By simulation it turns out that the trade-off curves, which show the relation between the expected number of rounds and the expected loss of inefficiency, constructed for different numbers of players coincide with each other. Thus, in expectation the number of rounds of the auction that obtains a desired level of efficiency is independent of the number of players.

The paper is organized as follows. Section 2 introduces the rules of the $c$-bisection auction. In Section 3 the running time of the auction is analyzed. Section 4 is devoted to the analysis of the efficiency of the auction. Concluding remarks about the trade-off are reported in Section 5. The Appendix contains proofs of some statements and tables with computational results.

\section{The $c$-bisection auction}

Suppose a single indivisible object is auctioned to a set $N=\{1, \ldots, n\}$ of players. The players have independent private valuations, $v_{i}$, drawn from a common continuous probability distribution with cumulative density $F(v)$, within the range $[\alpha, \beta)$. We assume quasi-linear utilities. Valuations of players are private information, i.e. each player knows only his own valuation but not the valuations of the others. Before the start of the auction there is a lottery that determines the order of the players. W.l.o.g. we assume that this ordering is $1 \prec 2 \prec 3 \prec \cdots \prec n-1 \prec n$. A player with a lower ranking is called a predecessor. So e.g. player 5 is a predecessor of player 7 .

The auction runs for an a priori indefinite number of rounds. Each round is characterized by payment $p_{r}$, query price $q_{r}$, upper bound $u_{r}$ and a set of active players $A_{r}$. The payment specifies the price to be payed if a player wins in this round. The query price is used by the auctioneer to ask an active player whether his valuation is larger than or equal to the query price. Players are queried openly in increasing order, so that a player can observe the bids of his predecessors. In each round the query price $q_{r}$ is chosen from the open interval $\left(p_{r}, u_{r}\right)$.

The initial set of active players is $A_{1}=N$. The auction starts with $p_{1}=\alpha$ and $u_{1}=\beta$ and $q_{1}$ is a point in $(\alpha, \beta)$. Given the current set $A_{r}$, the payment $p_{r}$, the query price $q_{r}$, the upper bound $u_{r}$ and the bids of players in round $r$ the characteristics of the next round $r+1$ are defined as follows. If all players submit a no bid they all remain active, i.e. $A_{r+1}=A_{r}$. The payment remains the same and the upper bound is set to the previous query price, i.e. $p_{r+1}=p_{r}$ and $u_{r+1}=q_{r}$. If at least two players submit a 
yes bid, all players that said yes remain active. The upper bound remains the same and the payment is set equal to the previous query price, i.e. $u_{r+1}=u_{r}$ and $p_{r+1}=q_{r}$. The new bounds determine a new query price $q_{r+1}$ in $\left(p_{r+1}, u_{r+1}\right)$. If only one player submits a yes bid the auction stops, this player wins the auction and pays $p_{r}$. If such a moment doesn't occur, i.e. at least two players remain always active, the winner is determined according to the order of players: among those players who remain active the player with the highest ranking wins. The price the winner pays is equal to the limit of the sequence of the payments that occurred in the subsequent rounds in the auction. Since the sequence of payments is increasing this limit is equal to the supremum of the payments.

The query price is defined as follows. For a given $c \in(0,1)$ and any continuous probability distribution with cumulative density $F(v)$ from which valuations of players are drawn, in any round $r$, given the payment $p_{r}$ and the upper bound $u_{r}$, the query price $q_{r}$ is chosen such that

$$
\frac{F\left(q_{r}\right)-F\left(p_{r}\right)}{F\left(u_{r}\right)-F\left(p_{r}\right)}=c,
$$

i.e. interval $\left[p_{r}, q_{r}\right)$ contains a fraction $c$ of the measure of $\left[p_{r}, u_{r}\right)$. For example, for uninform distribution the query price bisects the interval $\left[p_{r}, u_{r}\right)$ in fractions $c$ and $1-c$ so that $q_{r}=p_{r}+c\left(u_{r}-p_{r}\right)$.

The following strategy, called the bluff strategy, constitutes a symmetric ex-post equilibrium in the $c$ bisection auction (this result is proven in [6]). An ex-post equilibrium is a strategy profile such that, given any realization of valuations, the plan of actions prescribed by the strategy to a player is a best response to the plans of actions prescribed by the strategies of the other players. Under the bluff strategy an active player $i$ having valuation $v_{i}$ says yes in round $r$ whenever:

1. $v_{i} \geq q_{r}$, or

2. $p_{r} \leq v_{i}<q_{r}$ and no active predecessor of him said yes in this round.

The following example illustrates how the auction proceeds under the bluff equilibrium.

Example. Suppose five players with valuations uniformly distributed on $[0,1)$ participate in the $c$ bisection auction with $c$ equal to 0.5 . Suppose that according to the lottery the ordering of players is $A \prec B \prec C \prec D \prec E$. Players have the following private valuations respectively: 0.43, 0.71, 0.38, 0.79, and 0.86 . The auction proceeds as follows:

\begin{tabular}{llllccccc}
\hline Round & Payment & Query price & Set of active & Player & Player & Player & Player & Player \\
$r$ & $p_{r}$ & $q_{r}$ & players $A_{r}$ & A & B & C & D & E \\
\hline 1 & 0 & 0.5 & ABCDE & yes & yes & no & yes & yes \\
2 & 0.5 & 0.75 & ABDE & no & yes & - & yes & yes \\
3 & 0.75 & 0.875 & BDE & - & no & - & yes & no \\
\hline
\end{tabular}


In the first round player $\mathrm{A}$, having no predecessor and valuation larger than $p_{1}$ says yes. Every other player, having predecessor A with yes decision, says yes iff his valuation is larger than $q_{1}=0.5$. All players except $\mathrm{C}$ say yes and therefore remain active. The payment and the query price increase to 0.5 and 0.75 , respectively. Since $v_{A}<p_{2}$ player A says no in the second round. Now player B has no predecessor with yes decision and since $v_{B}>p_{2}$ he says yes. Players $\mathrm{D}$ and E say yes since their valuations are larger than $q_{2}=0.75$. Again the payment and the query price increase. In the third round player B says no, player $D$, having now no predecessors with yes decision, says yes and player $\mathrm{E}$ says no. So there is only one yes decision meaning that the auction ends. Player D wins the auction and pays 0.75 .

Notice that the outcome in the example is not efficient - the winner is not the player with the highest valuation. But as we have already pointed out inefficiency for some realizations of valuations is inevitable. Later in the paper we investigate how inefficient this auction is by analyzing the probability of inefficient allocation and the expected loss of welfare.

Probability distribution of player actions. In the remaining part of the paper we focus on auction performance in expectation. In order to analyze the expected performance we need to know the probability of particular actions of players. Namely, we need to know the probability of saying yes and no by an active player under the bluff strategy.

Recall that in any round $r$ of the $c$-bisection auction the query price $q_{r}$ is determined in such a way that, given that the valuation of a player is in $\left[p_{r}, u_{r}\right)$, the probability that his valuation is in $\left[p_{r}, q_{r}\right)$ is equal to $c$. Write $i_{r}:=\min \left\{i \mid i \in A_{r}\right\}$ - among the active players in round $r$ the one with the lowest ranking; $j_{r}:=\min \left\{i \mid i \in A_{r}, i \neq i_{r}\right\}$ - among the active players in round $r$ the one with the second lowest ranking.

First let's observe that when player $i_{r}$ says no for the first time, player $j_{r}$ says yes with certainty. Indeed, in all previous rounds player $i_{r}$ said yes and since $j_{r}$ is active in round $r$ also he said yes in those rounds. Both the payment and the query price increased so that $p_{r}=q_{r-1}$. Since player $j_{r}$ follows the bluff strategy his previous yes decision implies that $v_{j_{r}} \geq q_{r-1}=p_{r}$. If in round $r$ player $i_{r}$ says no, player $j_{r}$ is in the situation where he doesn't have any active predecessor with yes decision and therefore says yes whenever his valuation is not smaller than $p_{r}$, that is with certainty. It follows that after round $r$ player $i_{r}$ drops out so that $i_{r+1}=j_{r}$. Notice that in the equilibrium in every round $r$ either player $i_{r}$ or player $j_{r}$ (or both) say yes. It means that in the equilibrium only players with yes decision remain active. This in turn implies that the upper bound always remains the same so that $u_{r}=\beta$ for any $r$, and the payment and the query price increase, so that $p_{r}=q_{r-1}$ for any $r>1$. 
Second, we need to know the probability that player $i_{r}$ says yes in round $r$. Having no active predecessor player $i_{r}$ says yes iff $v_{i_{r}} \geq p_{r}$. Since $p_{1}=\alpha$ player $i_{1}$ in round 1 says yes with certainty. Now let's show that for any $r>1$ the probability that player $i_{r}$ says yes in round $r$ equals $1-c$. Regarding the identity of player $i_{r}$ there are two possibilities - either $i_{r}=i_{r-1}$ (happens if decision of $i_{r-1}$ was yes) or $i_{r}=j_{r-1}$ (happens if decision of $i_{r-1}$ was no and consequently decision of $j_{r-1}$ was yes). In both cases the decision of player $i_{r}$ in round $r-1$ was yes implying that $v_{i_{r}} \geq p_{r-1}$. Thus, $P\left(v_{i_{r}} \geq p_{r} \mid v_{i_{r}} \geq\right.$ $\left.p_{r-1}\right)=P\left(v_{i_{r}} \geq q_{r-1} \mid v_{i_{r}} \geq p_{r-1}\right)=1-c$. The last equality holds because $q_{r-1}$ divides the interval $\left(p_{r-1}, \beta\right)$ exactly in such a way that this conditional probability is equal to $1-c$.

Further, we need to know the probability of saying yes in round $r$ for any player $i \neq i_{r}, i \in A_{r}$. We can distinguish two cases. First, consider the case where player $i$ says yes in round $r$. From the fact that $i \in A_{r}$ follows that player $i_{r}$ said yes in round $r-1$ and thus $v_{i} \geq q_{r-1}$. In round $r$ he says yes iff $v_{i} \geq q_{r}$. Thus, $P\left(v_{i} \geq q_{r} \mid v_{i} \geq q_{r-1}\right)=P\left(v_{i} \geq q_{r} \mid v_{i} \geq p_{r}\right)=1-c$. The last equality holds because $q_{r}$ divides the interval $\left(p_{r}, \beta\right)$ exactly in such a way that this conditional probability is equal to $1-c$. Secondly, consider the case where player $i_{r}$ says no in round $r$. As we described above player $j_{r}$ says yes with certainty. For any other player $i$ the situation is the same as in the previous case and thus also here player $i \in N \backslash\left\{i_{r}, j_{r}\right\}$ says yes with probability $1-c$.

Now notice that the analysis above was done without specifying the distribution function from which valuations of players are drawn. Due to the price setting rule of the $c$-fraction auction the obtained probability results hold regardless of the distribution function of valuations. Thus, in the remaining part of the paper, namely in Section 4, for simplicity of argumentation we focus on the setting where valuations of players are independently drawn from the uniform distribution in $[0,1)$. Moreover, it could be seen from the analysis above that the probability of saying yes or no by an active player doesn't depend on the round. It enables us to derive recursive formulas (in the number of active players) for the expected number of rounds and the expected number of queries performed in the auction.

\section{Running time of the auction}

In this section we investigate the expected running time of the $c$-bisection auction if the bluff strategies are played. We analyze two measures, namely the expected number of rounds and the expected number of queries performed in the auction before the winner is found. As a query we consider each separate question of the auctioneer to an active player. As a round we consider a sequence of queries in which each active player is asked to act exactly once. For both measures we derive first a recursive formula and give then an upper bound for the function defined by this formula. 


\subsection{The expected number of rounds}

Let $e_{c}(k)$ be the expected number of rounds of the auction with $k$ active players, given that the decision of the active player with the lowest ranking is yes in the current round; and $e_{c}^{*}(k)$ be the expected number of rounds given that this decision is no. Consider round $r$ with $n$ active players and suppose that the decision of player $i_{r}$ in the current round is yes. The current round contributes 1 to $e_{c}(n)$. Now let's compute the expected number of remaining rounds. If all active players apart from player $i_{r}$ say $n o$, the auction stops after this round. If $k$ (for some $1 \leq k \leq n-1$ ) active players apart from player $i_{r}$ say yes, then the auction continues with $k+1$ active players. The probability of this situation given the yes decision of player $i_{r}$ is $\left(\begin{array}{c}n-1 \\ k\end{array}\right)(1-c)^{k} c^{n-1-k}$ (since when player $i_{r}$ says yes all other players say yes and no with probabilities $1-c$ and $c$ respectively). In the next round player $i_{r+1}=i_{r}$ says yes or no with probabilities $1-c$ and $c$ respectively. Thus if $k$ active players apart from player $i_{r}$ say yes in the round $r$, the expected number of remaining rounds is equal to $(1-c) e_{c}(k+1)+c e_{c}^{*}(k+1)$. Hence, for any $n \geq 2$

$$
e_{c}(n)=1+\sum_{k=1}^{n-1}\left(\begin{array}{c}
n-1 \\
k
\end{array}\right)(1-c)^{k} c^{n-1-k}\left[(1-c) e_{c}(k+1)+c e_{c}^{*}(k+1)\right] .
$$

Now recall that if player $i_{r+1}$ says no player $j_{r+1}$ says yes with certainty, which causes player $i_{r+1}$ to drop out of the auction. Thus, $e_{c}^{*}(2)=1$ and $e_{c}^{*}(k+1)=e_{c}(k)$ for any $k>1$. These observations are used in Appendix to rewrite the above recursive relation to

$$
\left[1-(1-c)^{n}\right] e_{c}(n)=1+(n-1)(1-c) c^{n-1}+\sum_{k=2}^{n-1}\left(\begin{array}{l}
n \\
k
\end{array}\right)(1-c)^{k} c^{n-k} e_{c}(k) .
$$

This formula is valid for any $n \geq 2$.

Now notice that since in the first round player $i_{1}$ says yes with certainty, the expected number of rounds of the auction of $n$ players is equal to $e_{c}(n)$. Thus using formula 2 we can compute the expected number of rounds in the auction of $n$ players. Plugging in $n=2$ yields $e_{c}(2)=\frac{1+c(1-c)}{c(2-c)}$. All other values can be determined recursively. Table 1 in Appendix presents the computational results for different values of $c$ in the auction with up to 100 players (data is within an accuracy of 0.001). Figure 1(a) shows how for a fixed value of $c$ the expected number of rounds increases in the number of players who participate in the auction. Furthermore, Figure 1(b) demonstrates how for a fixed number of players the expected number of rounds decreases as $c$ increases.

Generally we show that the expected number of rounds of the auction is bounded from above by a function that is logarithmic in the number of players. To prove this, first we introduce several notations and lemmas. 


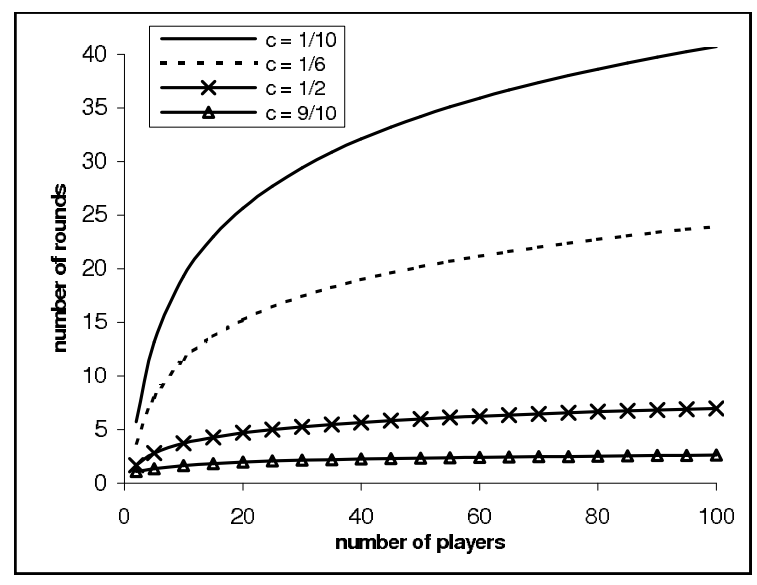

a)

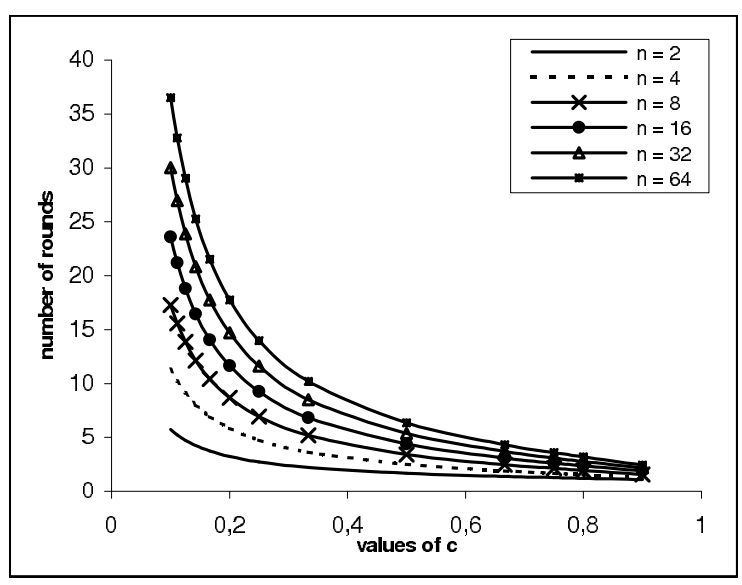

b)

Figure 1: The expected number of rounds (a) for different fixed values of $c$; (b) for different fixed numbers of players.

Define $D_{n}=\prod_{k=1}^{n} \frac{1}{1-(1-c)^{k}}$ for any $n \geq 2$.

Also define $E_{2}=\frac{1+c(1-c)}{c(2-c)}$ and for any $n>2$

$$
E_{n}=1+(n-1)(1-c) c^{n-1}+\sum_{k=2}^{n-1}\left(\begin{array}{l}
n \\
k
\end{array}\right)(1-c)^{k} c^{n-k} E_{k} .
$$

Lemma 3.1 For any $n \geq 2$, $e(n) \leq E_{n} \cdot D_{n}$.

Proof. The proof is by induction on $n$. The basis of the induction is trivial since $e_{c}(2)=E_{2}$ and $D_{2}>1$. Suppose that $e_{c}(k) \leq E_{k} \cdot D_{k}$ is true for any $2 \leq k \leq n-1$. Notice that $D_{n} \geq D_{n-1} \geq \ldots \geq D_{2}>1$. Thus, using the recursive formula for $e_{c}(n)$ and the induction hypothesis,

$$
\begin{aligned}
{\left[1-(1-c)^{n}\right] e_{c}(n) } & =1+(n-1)(1-c) c^{n-1}+\sum_{k=2}^{n-1}\left(\begin{array}{l}
n \\
k
\end{array}\right)(1-c)^{k} c^{n-k} e_{c}(k) \\
& \leq 1+(n-1)(1-c) c^{n-1}+\sum_{k=2}^{n-1}\left(\begin{array}{l}
n \\
k
\end{array}\right)(1-c)^{k} c^{n-k} E_{k} D_{k} \\
& \leq 1+(n-1)(1-c) c^{n-1}+\sum_{k=2}^{n-1}\left(\begin{array}{l}
n \\
k
\end{array}\right)(1-c)^{k} c^{n-k} E_{k} D_{n-1} \\
& \leq D_{n-1}\left[1+(n-1)(1-c) c^{n-1}+\sum_{k=2}^{n-1}\left(\begin{array}{l}
n \\
k
\end{array}\right)(1-c)^{k} c^{n-k} E_{k}\right] \\
& =E_{n} \cdot D_{n-1},
\end{aligned}
$$

which completes the proof. 
Now we find bounds on $D_{n}$ and $E_{n}$.

Lemma 3.2 For any $n \geq 2, D_{n} \leq e^{\frac{1-c}{c^{2}}}$.

Proof. It's enough to show that $\ln D_{n} \leq \frac{1-c}{c^{2}}$. Let's define $\lambda=\frac{1}{1-c}$. Notice that since $0<c<1$ it holds that $\lambda>1$.

We have

$$
\begin{aligned}
\ln D_{n} & =\ln \left(\prod_{k=1}^{n} \frac{\lambda^{k}}{\lambda^{k}-1}\right)=\sum_{k=1}^{n}\left[\ln \lambda^{k}-\ln \left(\lambda^{k}-1\right)\right] \leq \sum_{k=1}^{n}(\ln x)_{\mid x=\lambda^{k}-1}^{\prime}=\sum_{k=1}^{n} \frac{1}{\lambda^{k}-1} \\
& \leq \sum_{k=1}^{n} \frac{1}{\lambda^{k}-\lambda^{k-1}}=\frac{1}{\lambda-1} \sum_{k=1}^{n} \frac{1}{\lambda^{k-1}} \leq \frac{1}{\lambda-1} \sum_{k=0}^{\infty} \frac{1}{\lambda^{k}}=\frac{\lambda}{(\lambda-1)^{2}}=\frac{1-c}{c^{2}} .
\end{aligned}
$$

Lemma 3.3 For any $n \geq 2$ and any $c \leq \frac{1}{2}, E_{n} \leq 1+\log _{a} n$, with base $a=\frac{1}{1-c}$.

Proof. The proof is by induction on $n$. The basis of induction holds since $\frac{1+c(1-c)}{c(2-c)} \leq \log _{a} 2+1$ for any $c \leq \frac{1}{2}$. Suppose $E_{k} \leq 1+\log _{a} k$ for any $2 \leq k \leq n-1$. Using the induction hypothesis,

$$
\begin{aligned}
E_{n} & =1+(n-1)(1-c) c^{n-1}+\sum_{k=2}^{n-1}\left(\begin{array}{l}
n \\
k
\end{array}\right)(1-c)^{k} c^{n-k} E_{k} \\
& \leq 1+(n-1)(1-c) c^{n-1}+\sum_{k=2}^{n-1}\left(\begin{array}{l}
n \\
k
\end{array}\right)(1-c)^{k} c^{n-k}\left(\log _{a} k+1\right) \\
& \leq 1+\sum_{k=2}^{n-1}\left(\begin{array}{l}
n \\
k
\end{array}\right)(1-c)^{k} c^{n-k} \log _{a} k+\sum_{k=1}^{n-1}\left(\begin{array}{l}
n \\
k
\end{array}\right)(1-c)^{k} c^{n-k} \\
& \leq 2+\sum_{k=2}^{n-1}\left(\begin{array}{l}
n \\
k
\end{array}\right)(1-c)^{k} c^{n-k} \log _{a} k .
\end{aligned}
$$

Since the logarithm with base $a=\frac{1}{1-c}$ is concave, we know that if $\lambda_{k} \geq 0$ and $\sum_{k=0}^{n} \lambda_{k}=1$ then

$$
\sum_{k=0}^{n} \lambda_{k} \log _{a}\left(x_{k}\right) \leq \log _{a}\left(\sum_{k=0}^{n} \lambda_{k} x_{k}\right) .
$$

So let's take $\lambda_{k}=\left(\begin{array}{l}n \\ k\end{array}\right)(1-c)^{k} c^{n-k}$ for all $k$ and take $x_{0}=x_{n}=1, x_{k}=k$ for any $1 \leq k \leq n-1$. Then

$$
\begin{aligned}
E_{n} & \leq 2+\sum_{k=2}^{n-1}\left(\begin{array}{l}
n \\
k
\end{array}\right)(1-c)^{k} c^{n-k} \log _{a} k \\
& =2+\sum_{k=0}^{n}\left(\begin{array}{l}
n \\
k
\end{array}\right)(1-c)^{k} c^{n-k} \log _{a}\left(x_{k}\right) \\
& \leq 2+\log _{a}\left[\sum_{k=0}^{n}\left(\begin{array}{l}
n \\
k
\end{array}\right)(1-c)^{k} c^{n-k} x_{k}\right] \\
& =2+\log _{a}\left[\sum_{k=1}^{n-1}\left(\begin{array}{l}
n \\
k
\end{array}\right)(1-c)^{k} c^{n-k} k+c^{n}+(1-c)^{n}\right]
\end{aligned}
$$




$$
\begin{aligned}
& \leq 2+\log _{a}\left[\sum_{k=1}^{n-1}\left(\begin{array}{l}
n \\
k
\end{array}\right)(1-c)^{k} c^{n-k} k+n(1-c)^{n}\right] \\
& =2+\log _{a}\left[\sum_{k=0}^{n}\left(\begin{array}{l}
n \\
k
\end{array}\right)(1-c)^{k} c^{n-k} k\right] \\
& =2+\log _{a}[(1-c) n] \\
& =1+\log _{a} n .
\end{aligned}
$$

The last inequality holds since for any $c \leq \frac{1}{2}$ and any $n \geq 2$ it holds that $c^{n}+(1-c)^{n} \leq 2(1-c)^{n} \leq$ $n(1-c)^{n}$.

A final immediate consequence of Lemmas $3.1-3.3$ is the following theorem.

Theorem 3.4 For any $c \leq \frac{1}{2}$ and any $n \geq 2, e_{c}(n) \leq e^{\frac{1-c}{c^{2}}}\left(\log _{\frac{1}{1-c}} n+1\right)$.

Remark: Since $e_{c}(n)>e_{\bar{c}}(n)$ when $c<\bar{c}$, the upper bound for $c=\frac{1}{2}$ is also valid for any $c>\frac{1}{2}$.

We showed that the expected number of rounds of the $c$-bisection auction is bounded from above by a function that is logarithmic in the number of players. A comparison of the bound with the computed results suggests that this bound is not tight. It can be easily checked that for a fixed value of $c$ the ratio between the bound and the computed result is approximately constant (as a function of $n$ ), implying that the bound is likely to have the correct order of magnitude.

\subsection{The expected number of queries}

Let $b_{c}(k)$ be the expected number of queries of the auction with $k$ active players, given that the decision of the active player with the lowest ranking is yes in the current round; $b_{c}^{*}(k)$ be the expected number of queries given that this decision is no. Notice that in a round with $k$ active players $k$ queries are performed. Following the same argumentation as we used for determining the formula for the expected number of rounds we find that for any $n \geq 2$

$$
b_{c}(n)=n+\sum_{k=1}^{n-1}\left(\begin{array}{c}
n-1 \\
k
\end{array}\right)(1-c)^{k} c^{n-1-k}\left[(1-c) b_{c}(k+1)+c b_{c}^{*}(k+1)\right] .
$$

Again, notice that when player $i_{r+1}=i_{r}$ says no player $j_{r+1}$ says yes with certainty, which causes player $i_{r+1}$ to drop out of the auction. Thus, $b_{c}^{*}(2)=2$ and for all $k>1$ it holds that $b_{c}^{*}(k+1)=1+b_{c}(k)$.

This is used in Appendix to derive the following recursive relation. For any $n \geq 2$

$$
\left[1-(1-c)^{n}\right] b_{c}(n)=n+(n-1)(1-c) c^{n-1}+c-c^{n}+\sum_{k=2}^{n-1}\left(\begin{array}{l}
n \\
k
\end{array}\right)(1-c)^{k} c^{n-k} b_{c}(k)
$$




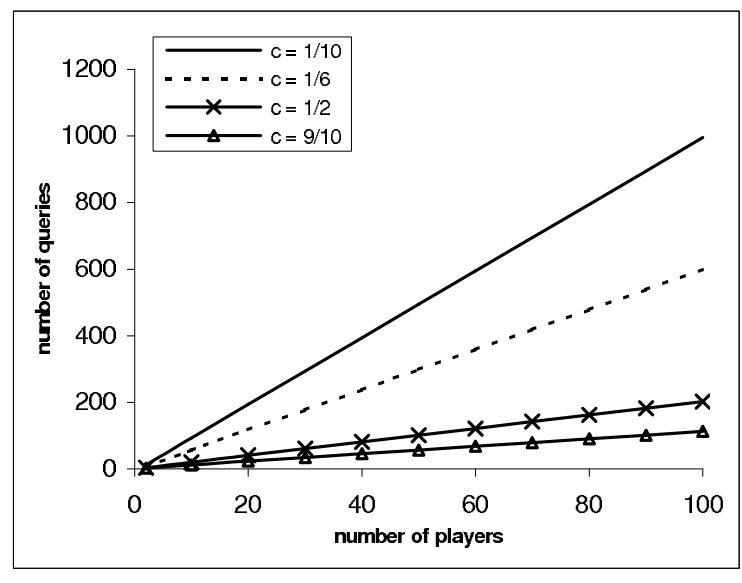

a)

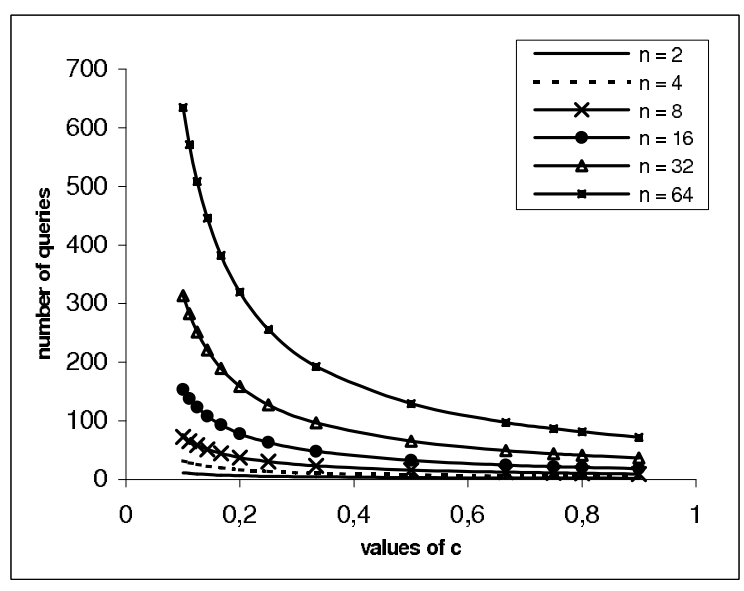

b)

Figure 2: The expected number of queries (a) for different fixed values of $c$; (b) for different fixed numbers of players.

Now notice that since in the first round player $i_{1}$ says yes with certainty, the expected number of queries in the auction of $n$ players is equal to $b_{c}(n)$. Thus using formula 4 we can compute the expected number of queries performed in the auction of $n$ players. Plugging in $n=2$ yields $b_{c}(2)=\frac{2+2 c(1-c)}{c(2-c)}$. All other values can be determined recursively. Table 2 in Appendix presents the computational results for different values of $c$ in the auction with up to 100 players (data is within an accuracy of 0.001). Figure 2(a) demonstrates that for a fixed value of $c$ the expected number of queries increases in the number of players participating in the auction. Figure 2(b) shows that for a fixed number of players the expected number of queries decreases as $c$ becomes larger.

Generally we show that the expected number of queries is bounded from above by a function that is linear in the number of players. To prove this we introduce several notations and lemmas.

Define $B_{2}=\frac{2+2 c(1-c)}{c(2-c)}$ and

$$
B_{n}=n+(n-1)(1-c) c^{n-1}+c-c^{n}+\sum_{k=2}^{n-1}\left(\begin{array}{l}
n \\
k
\end{array}\right)(1-c)^{k} c^{n-k} B_{k}
$$

for any $n>2$.

Recall that $D_{n}=\prod_{k=1}^{n} \frac{1}{1-(1-c)^{k}}$.

Lemma 3.5 For any $n \geq 2, b_{c}(n) \leq B_{n} \cdot D_{n}$.

Proof. The proof is identical to the proof of Lemma 3.1 if we replace $e_{c}(k)$ by $b_{c}(k)$ and $E_{k}$ by $B_{k}$ for all $2 \leq k \leq n$. 
From Lemma 3.2 we know that for any $n \geq 2, D_{n} \leq e^{\frac{1-c}{c^{2}}}$. Now we find a bound on $B_{n}$.

Lemma 3.6 For any $n \geq 2, B_{n} \leq\left(\frac{2}{c}+\frac{1}{2}\right)(n+1)$.

Proof. The proof is by induction on $n$. The basis of the induction holds since it can be easily shown that $B_{2}<3\left(\frac{2}{c}+\frac{1}{2}\right)$. Now suppose that $B_{k} \leq\left(\frac{2}{c}+\frac{1}{2}\right)(k+1)$ for any $2 \leq k \leq n-1$. Using the induction hypothesis,

$$
\begin{aligned}
B_{n} & =n+(n-1)(1-c) c^{n-1}+c-c^{n}+\sum_{k=2}^{n-1}\left(\begin{array}{l}
n \\
k
\end{array}\right)(1-c)^{k} c^{n-k} B_{k} \\
& \leq n+(n-1)(1-c) c^{n-1}+c-c^{n}+\sum_{k=2}^{n-1}\left(\begin{array}{l}
n \\
k
\end{array}\right)(1-c)^{k} c^{n-k}\left(\frac{2}{c}+\frac{1}{2}\right)(k+1) \\
& \leq 2 n+c+\left(\frac{2}{c}+\frac{1}{2}\right) \sum_{k=0}^{n}\left(\begin{array}{l}
n \\
k
\end{array}\right)(1-c)^{k} c^{n-k} k+\left(\frac{2}{c}+\frac{1}{2}\right) \sum_{k=0}^{n}\left(\begin{array}{l}
n \\
k
\end{array}\right)(1-c)^{k} c^{n-k} \\
& =2 n+c+\left(\frac{2}{c}+\frac{1}{2}\right)(1-c) n+\left(\frac{2}{c}+\frac{1}{2}\right) \\
& =\left(\frac{2}{c}+\frac{1}{2}\right)(n+1)+c\left(1-\frac{n}{2}\right) \\
& \leq\left(\frac{2}{c}+\frac{1}{2}\right)(n+1) .
\end{aligned}
$$

The last inequality holds since $n \geq 2$.

A final immediate consequence of Lemmas 3.2, 3.5 and 3.6 is the following theorem.

Theorem 3.7 For any integer $n \geq 2, b_{c}(n) \leq e^{\frac{1-c}{c^{2}}}\left(\frac{2}{c}+\frac{1}{2}\right)(n+1)$.

We showed that the expected number of queries is bounded from above by a function that is linear in the number of players. Again, a comparison of the bound with the computed results suggests that this bound is not tight. It can be easily checked that for a fixed value of $c$ the ratio between the bound and the computed result is approximately constant (as a function of $n$ ), implying that the bound is likely to have the correct order of magnitude.

\section{Efficiency of the auction}

In this section we investigate the efficiency of the $c$-bisection auction when the bluff equilibrium is played. In particular we compute the probability of inefficient allocation and the expected loss of welfare. Here for simplicity of argumentation we focus on the setting where valuations of players are independently drawn from the uniform distribution in $[0,1)$. 
In order to compute these measures of inefficiency it is convenient to consider the direct revelation mechanism associated with the bluff equilibrium. We construct a direct auction that mimics the bluff strategies of the $c$-bisection auction.

\subsection{The direct $c$-bisection auction}

Consider the following direct auction $\left(w_{d}, p_{d}\right)$, called the direct $c$-bisection auction. For $r \in \mathbb{N}$, write $I_{r}:=\left[1-(1-c)^{r-1}, 1-(1-c)^{r}\right) .{ }^{6}$ Note that the intervals $I_{1}, I_{2}, \ldots$ partition the unit interval $[0,1)$ from which valuations are drawn. Now let $v=\left(v_{i}\right)_{i \in N}$ be a profile of valuations. Write $I_{r}(v):=I_{r} \cap\left\{v_{i} \mid\right.$ $i \in N\}$ - the set of valuations that belong to the interval $I_{r}$. Let $r(v)$ be the highest natural number $r$ for which $I_{r}(v)$ is not empty. Among players whose valuations belong to the interval $I_{r(v)}$ the one with the lowest ranking is declared to be the winner. So the winner $w_{d}$ is defined by

$$
w_{d}(v):=\min \left\{i \in N \mid v_{i} \in I_{r(v)}\right\}
$$

Let $s(v)$ be the highest natural number $r$ for which $I_{r} \cap\left\{v_{i} \mid i \in N \backslash\left\{w_{d}(v)\right\}\right\}$ is not empty. The price the winner pays is equal to the lower bound of interval $I_{s(v)}$ if all players whose valuations belong to this interval have a ranking higher than the winner. Otherwise the price equals the upper bound of this interval. So the payment $p_{d}$ is defined by

$$
p_{d}(v):= \begin{cases}1-(1-c)^{s(v)-1} & \text { if } i>w_{d}(v) \text { for all } i \in I_{s(v)}(v) \\ 1-(1-c)^{s(v)} & \text { else. }\end{cases}
$$

Notice that the first condition always holds if $\left|I_{r(v)}\right|>1$, i.e. if $s(v)=r(v)$. If $I_{r(v)}$ contains only one valuation, the payment depends on the ranking of the players with valuations in $I_{s(v)}$.

Example. Consider the same example as in Section 2 with players $A \prec B \prec C \prec D \prec E$ whose valuations are $0.43,0.71,0.38,0.79$ and 0.86 , respectively. Suppose that in the direct $c$-bisection auction with $c=1 / 2$ the players truthfully report their valuations. Then $r(v)=s(v)=3$. Players with valuation in $I_{3}$ are players $\mathrm{D}$ and E. Player $D$ has ranking lower than player $E$ so he is the winner. The price he pays for the object is equal to the lower bound of $I_{3}$, namely 0.75 . So we get the same outcome as the one we found in Section 2.

Generally, it is shown in [6] that for any realization of valuations $v=\left(v_{i}\right)_{i \in N}$ the outcome $\left(w_{d}(v), p_{d}(v)\right)$ equals the outcome of the $c$-bisection auction when players, having these valuations, follow the bluff strategies. Consequently, by the revelation principle [10] truth telling is a dominant strategy in the direct $c$-bisection auction. Due to this result the efficiency performance of both the $c$-bisection query

\footnotetext{
${ }^{6}$ In case of a general density function $F(v)$ in $[\alpha, \beta)$ intervals $I_{r}$ are defined recursively as follows. Write $I_{r}=\left[\alpha_{r}, \beta_{r}\right)$ where $\alpha_{1}=\alpha, \alpha_{r}=\beta_{r-1}$ and $\beta_{r}$ is chosen such that $\frac{F\left(\beta_{r}\right)-F\left(\alpha_{r}\right)}{F(\beta)-F\left(\alpha_{r}\right)}=c$.
} 


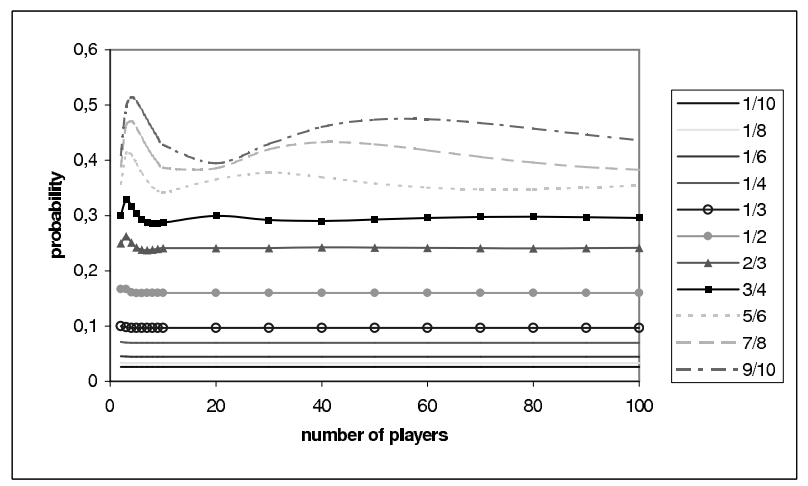

Figure 3: The probability of inefficient allocation.

auction under the bluff equilibrium and the direct $c$-bisection auction under the truth telling equilibrium are the same. Thus it suffices to compute the probability of inefficient allocation and the expected loss of welfare for the direct $c$-bisection auction under the truth telling equilibrium.

\subsection{The probability of inefficient allocation}

We derive a recursive formula for the probability of inefficient allocation and give an upper bound for the function defined by this formula. First notice that the direct $c$-bisection auction restricted to the interval $[c, 1)$ with $k$ players having valuations uniformly drawn from this interval has identical form and structure as the original direct auction with $k$ players having valuations uniformly drawn from $[0,1)$.

Let's denote by $P_{n}$ the probability that the auction with $n$ players terminates in an inefficient allocation. First, consider the case where the valuations of all players are smaller than $c$. The probability of this event is $c^{n}$. In this case the auction is only efficient if the player with the lowest ranking has the highest valuation. By symmetry this happens with probability $\frac{1}{n}$. Thus this case contributes $\frac{n-1}{n} c^{n}$ to $P_{n}$. Next consider the case where $k$ players have valuations larger than or equal to $c$ and $n-k$ players have valuations smaller than $c$. It happens with probability $\left(\begin{array}{l}n \\ k\end{array}\right) c^{n-k}(1-c)^{k}$. For $k=1$ the auction is efficient, so this case adds zero to $P_{n}$. For $k>1$ the auction can be inefficient and due to the structural similarity, inefficiency takes place with probability $P_{k}$. Hence,

$$
P_{n}=\frac{n-1}{n} c^{n}+\sum_{k=2}^{n}\left(\begin{array}{l}
n \\
k
\end{array}\right) c^{n-k}(1-c)^{k} P_{k} .
$$

This can be rewritten to the following recursive relation, $P_{2}=\frac{1}{2} \cdot \frac{c}{2-c}$ and for $n>2$ :

$$
\left[1-(1-c)^{n}\right] P_{n}=\frac{n-1}{n} c^{n}+\sum_{k=2}^{n-1}\left(\begin{array}{l}
n \\
k
\end{array}\right) c^{n-k}(1-c)^{k} P_{k}
$$


Direct computation of this expression for different combinations of $n$ and $c$ gives the values that are plotted in Figure 3. In general, we show the following upper bound on $P_{n}$.

Theorem 4.1 For all $n \geq 2, P_{n} \leq c$.

Proof. The proof is by induction on $n$. The basis of induction holds since $P_{2}=\frac{1}{2} \cdot \frac{c}{2-c} \leq c$. Suppose that $P_{k} \leq c$ for all $2 \leq k \leq n-1$. Then

$$
\begin{aligned}
P_{n} & =\frac{1}{1-(1-c)^{n}}\left[\frac{n-1}{n} \cdot c^{n}+\sum_{k=2}^{n-1}\left(\begin{array}{l}
n \\
k
\end{array}\right)(1-c)^{k} c^{n-k} \cdot P_{k}\right] \\
& \leq \frac{1}{1-(1-c)^{n}}\left[c^{n}+\sum_{k=2}^{n-1}\left(\begin{array}{l}
n \\
k
\end{array}\right)(1-c)^{k} c^{n-k} \cdot c\right] \\
& =\frac{1}{1-(1-c)^{n}}\left[c^{n}+c\left(1-c^{n}-n(1-c) c^{n-1}-(1-c)^{n}\right)\right] \\
& =\frac{c\left(1-(1-c)^{n}\right)}{1-(1-c)^{n}}+\frac{c^{n}-c^{n+1}-n(1-c) c^{n}}{1-(1-c)^{n}} \\
& =c+\frac{c^{n}(1-c)(1-n)}{1-(1-c)^{n}} \\
& \leq c .
\end{aligned}
$$

The first inequality holds by the induction assumption and the fact that $\frac{n-1}{n}<1$. The last inequality holds since $n \geq 2$.

Moreover, in the same way for $c \leq \frac{1}{2}$ it can be shown that $P_{n} \leq \frac{1}{2} c$ for all $n \geq 2$. This theorem shows in particular that by choosing an appropriate fraction $c$ in the auction we can make the probability of inefficiency as small as we like, independent of the number of players!

\subsection{The expected loss of welfare}

The welfare of an auction is equal to the valuation of the winner. Thus given a realization of valuations $v=\left(v_{i}\right)_{i \in N}$, the welfare achieved by the auction is the valuation of $w_{d}(v):=\min \left\{i \mid v_{i} \in I_{r(v)}\right\}$. The maximum welfare, given $v$, is $\max \left\{v_{i} \mid i \in N\right\}=\max \left\{v_{i} \mid v_{i} \in I_{r(v)}\right\}$. Thus, the loss $L(v)$ of welfare is

$$
L(v)=\max \left\{v_{i} \mid v_{i} \in I_{r(v)}\right\}-v_{w_{d}(v)}
$$

The expected loss of welfare, denoted by $L_{n}$, is the expected value of this difference. To estimate the value of $L_{n}$ we simulated the direct $c$-bisection auction and ran it for valuations uniformly and independently drawn from the interval [0,1). For each combination of value $c$ and number of players $n$ we ran 10000 trials. Figure 4 shows the $99 \%$ confidence interval for the expected loss of welfare. It is interesting to notice that the maximum expected loss doesn't arrive at the minimum number of players. 


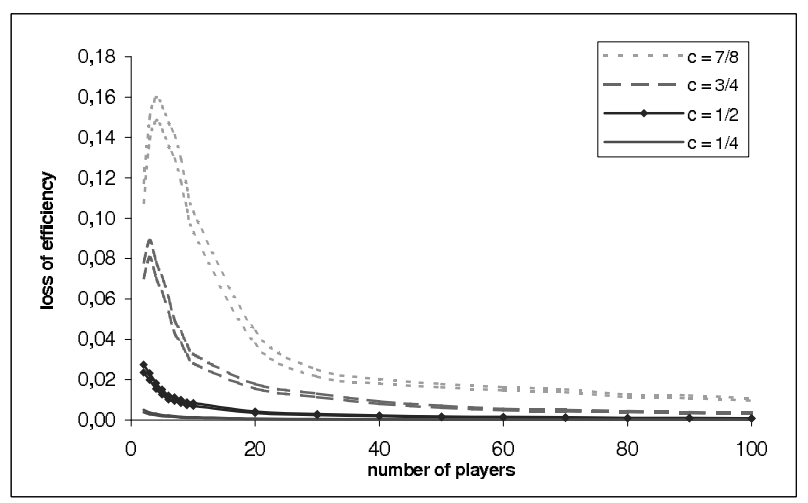

Figure 4: The expected loss of welfare, $99 \%$ confidence interval.

In general, we show the following statement. ${ }^{7}$

Theorem 4.2 For all $n \geq 2, L_{n} \leq c^{2}$.

Proof. Let $v=\left(v_{i}\right)_{i \in N}$ be a realization of valuations for which the allocation in the direct $c$-bisection auction is not efficient. In other words, $\max \left\{v_{i} \mid v_{i} \in I_{r(v)}\right\}>v_{w_{d}(v)}$. Since the valuation of $w_{d}(v)$ is an element of $I_{r(v)}$ we get that

$$
L(v) \leq \operatorname{length}\left(I_{r(v)}\right) \leq c .
$$

Hence, $L_{n} \leq c P_{n}$. Applying the result of Theorem 4.1 completes the proof.

As for probability of inefficient allocation, by choosing an appropriate fraction $c$ in the auction we can limit the expected loss of welfare to an arbitrary chosen level, independent of the number of players.

\section{Concluding remarks: trade-off between efficiency and run- ning time}

From the analysis above we derive the following relation between the value of $c$, the level of efficiency and the running time. For a fixed number of players, a smaller fraction $c$ leads to a lower expected loss of welfare and lower probability of inefficient allocation. But at the same time it leads to a higher expected number of rounds and queries. Thus, increasing running time is a price that we have to pay for increasing efficiency. Depending on the priorities of the auctioneer he may trade off efficiency against running time. Figure 5 shows, for some fixed $n$, the relation between the expected running time and the probability of inefficient allocation. These relations are built on computational results based on recursive formulas 2, 4 and 5. Figure 6 shows, for some fixed $n$, the relation between the expected running time

\footnotetext{
${ }^{7}$ This result can not be generalized to an arbitrary density function $F(v)$ since it's based on the lengths of intervals $I_{r}$ which entirely depend on distribution of valuations.
} 


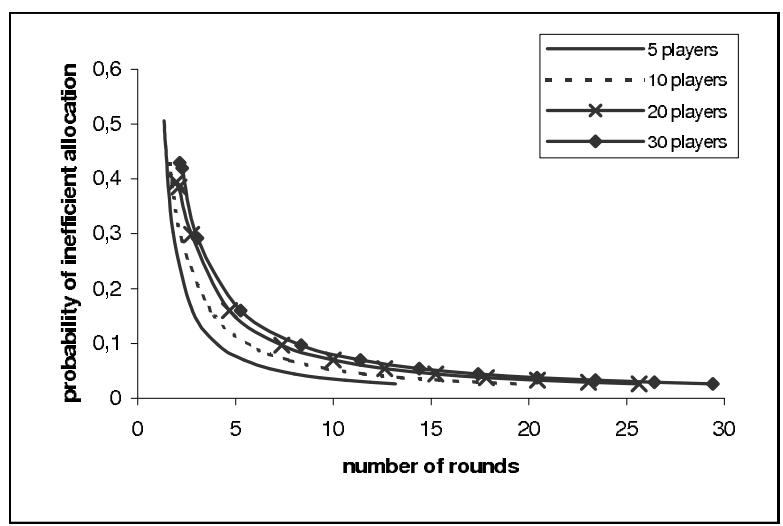

a)

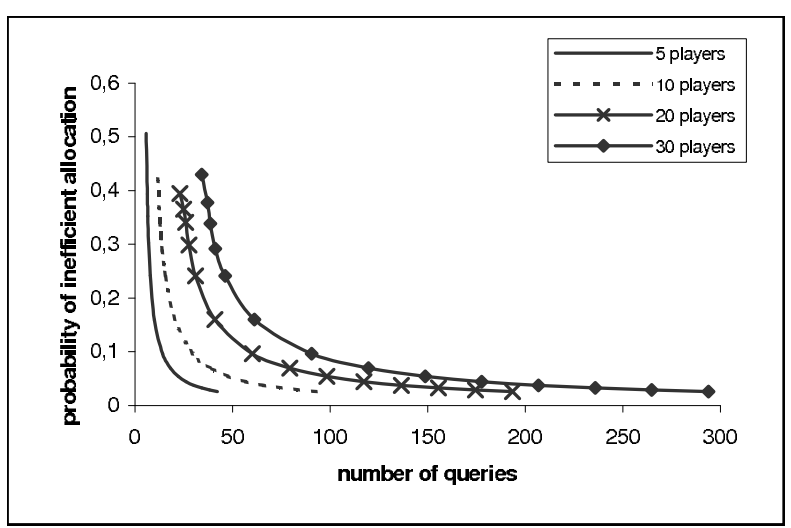

b)

Figure 5: The trade-off between (a) the probability of inefficient allocation and the number of rounds; (b) the probability of inefficient allocation and the number of queries.

and the expected loss of inefficiency. Because we don't have exact values for the expected loss of welfare we estimated the values by taking the middle point of the $99 \%$ confidence interval from the simulation results reported above. Notice that in Figure 6(a) the trade-off curves drawn for different numbers of players almost coincide with each other. It means that in order to get the desired level of efficiency we need to run the auction that in expectation takes the same number of rounds for any number of players participating in the auction (of course, the choice of $c$ to be used in this auction will depend on the number of players). This explains why for the same level of efficiency more players require more queries to be asked, which is demonstrated in Figure 6(b).

\section{References}

[1] L. Blumrosen, N. Nisan, and I. Segal. Multi-player and multi-round auctions with severely bounded communication. In Proccedings of 11th Annual Earopean Symposium on Algorithms (ESA 03), Budapest, Hungary, 2003.

[2] W. Conen and T. Sandholm. Preference elicitation in combinatorial auctions: Extended abstract. In Proceedings of the ACM Conference on Electronic Commerce (ACM-EC), Melbourne, Australia, 2001.

[3] V. Conitzer and T. Sandholm. Computational criticisms of the revelation principle. In Proceedings of the AAMAS-03 Workshop on Agent Mediated Electronic Commerce $V$ (AMEC V), Melbourne, Australia, 2003. 


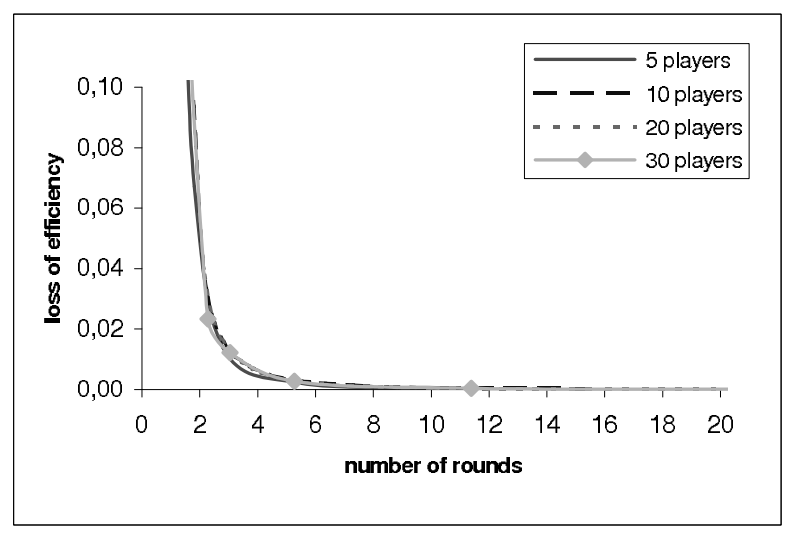

a)

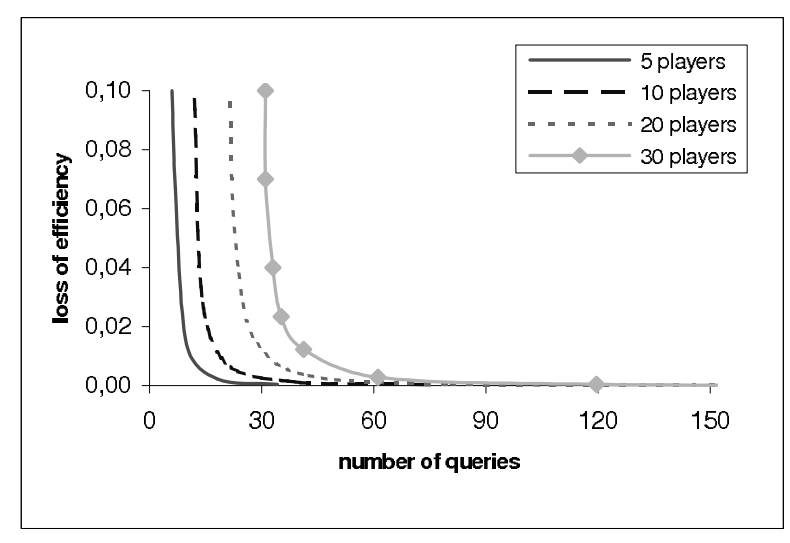

b)

Figure 6: The trade-off between (a) the loss of efficiency and the number of rounds; (b) the loss of efficiency and the number of queries.

[4] E. David, A. Rogers, J. Schiff, S. Kraus, and N. Jennings. Optimal design of English auctions with discrete bid levels. In Proceedings of ACM Conference on Electronic Commerce (EC'05), pages 98-107, Vancouver, Canada, 2005.

[5] E. Grigorieva, P.-J. Herings, R. Müller, and D. Vermeulen. The communication complexity of private value single item auctions. Operations Research Letters, 2006. to appear.

[6] E. Grigorieva, P.-J. Herings, R. Müller, and D. Vermeulen. Inefficiency of equilibria in query auctions with continuous valuations. METEOR Research Memorandum, Maastricht University, 2006.

[7] E. Grigorieva, P.-J. Herings, R. Müller, and D. Vermeulen. The private value single item bisection auction. Economic Theory, 2006. to appear.

[8] B. Hudson and T. Sandholm. Effectiveness of preference elicitation in combinatorial auctions. In Proceedings of the Agent-Mediated Electronic Commerce (AMEC) workshop at AAMAS-02, Bologna, Italy, 2004.

[9] K. Larson and T. Sandholm. Costly valuation computation in auctions. In Proceedings of the Theoretical Aspects of Reasoning about Knowledge (TARK), pages 169-182, Siena, Italy, 2001.

[10] A. Mas-Colell, M. Whinston, and J. Green. Microeconomic Theory. New York, Oxford University Press, 1995.

[11] N. Nisan and I. Segal. The communication requirements of efficient allocations and supporting prices. Journal of Economic Theory, 2005. to appear. 
[12] D. Parkes. Optimal auction design for agents with hard valuation problems. In Proccedings of IJCAI-99 Workshop on Agent Mediated Electronic Commerce, pages 206-219, Stockholm, Sweden, 1999.

[13] M. Rothkopf, T. Tisberg, and E. Kahn. Why are Vickrey auctions rare? Journal of Political Economy, 98:94-109, 1990.

[14] T. Sandholm. Issues in computational Vickrey auctions. International Journal of Electronic Commerce, 4.

[15] T. Sandholm and C. Boutilier. Preference elicitation in combinatorial auctions. In P. Cramton, Y. Shoham, and R. Steinberg, editors, Combinatorial Auctions. MIT Press, 2006. 


\section{Appendix}

\section{Derivation of formula 2}

Let's denote by $P_{k}^{n}=\left(\begin{array}{l}n \\ k\end{array}\right)(1-c)^{k} c^{n-k}$. Using the facts that $e_{c}^{*}(2)=1$ and $e_{c}^{*}(k+1)=e_{c}(k)$ for all $k \geq 2$ we can rewrite formula 1 as follows:

$$
\begin{aligned}
e_{c}(n) & =1+\sum_{k=1}^{n-1} P_{k}^{n-1}\left[(1-c) e_{c}(k+1)+c e_{c}^{*}(k+1)\right] \\
& =1+(1-c) \sum_{k=1}^{n-2} P_{k}^{n-1} e_{c}(k+1)+(1-c) P_{n-1}^{n-1} e_{c}(n)+c \sum_{k=2}^{n-1} P_{k}^{n-1} e_{c}^{*}(k+1)+c P_{1}^{n-1} e_{c}^{*}(2) \\
& =1+(1-c) \sum_{k=1}^{n-2} P_{k}^{n-1} e_{c}(k+1)+(1-c)^{n} e_{c}(n)+c \sum_{k=2}^{n-1} P_{k}^{n-1} e_{c}(k)+(n-1)(1-c) c^{n-1} \\
& =1+(1-c)^{n} e_{c}(n)+(n-1)(1-c) c^{n-1}+(1-c) \sum_{k=2}^{n-1} P_{k-1}^{n-1} e_{c}(k)+c \sum_{k=2}^{n-1} P_{k}^{n-1} e_{c}(k) \\
& =1+(1-c)^{n} e_{c}(n)+(n-1)(1-c) c^{n-1}+\sum_{k=2}^{n-1}\left[(1-c) P_{k-1}^{n-1}+c P_{k}^{n-1}\right] e_{c}(k) \\
& =1+(1-c)^{n} e_{c}(n)+(n-1)(1-c) c^{n-1}+\sum_{k=2}^{n-1} P_{k}^{n} e_{c}(k) .
\end{aligned}
$$

This can be rewritten to

$$
\left[1-(1-c)^{n}\right] e_{c}(n)=1+(n-1)(1-c) c^{n-1}+\sum_{k=2}^{n-1}\left(\begin{array}{l}
n \\
k
\end{array}\right)(1-c)^{k} c^{n-k} e_{c}(k) .
$$




\section{Derivation of formula 4}

Recall that $P_{k}^{n}=\left(\begin{array}{l}n \\ k\end{array}\right)(1-c)^{k} c^{n-k}$ Using the facts that $b^{*}(2)=2$ and $b^{*}(k+1)=b(k)+1$ for all $k \geq 2$, we get from formula 3 that

$$
\begin{aligned}
b_{c}(n)= & n+\sum_{k=1}^{n-1} P_{k}^{n-1}\left[(1-c) b_{c}(k+1)+c b_{c}^{*}(k+1)\right] \\
= & n+(1-c) \sum_{k=1}^{n-2} P_{k}^{n-1} b_{c}(k+1)+(1-c) P_{n-1}^{n-1} b_{c}(n)+c \sum_{k=2}^{n-1} P_{k}^{n-1} b_{c}^{*}(k+1)+c P_{1}^{n-1} b_{c}^{*}(2) \\
= & n+(1-c) \sum_{k=1}^{n-2} P_{k}^{n-1} b_{c}(k+1)+(1-c)^{n} b_{c}(n)+c \sum_{k=2}^{n-1} P_{k}^{n-1}\left[b_{c}(k)+1\right]+2(n-1)(1-c) c^{n-1} \\
= & n+(1-c)^{n} b_{c}(n)+2(n-1)(1-c) c^{n-1}+(1-c) \sum_{k=2}^{n-1} P_{k-1}^{n-1} b_{c}(k)+c \sum_{k=2}^{n-1} P_{k}^{n-1} b_{c}(k)+c \sum_{k=2}^{n-1} P_{k}^{n-1} \\
= & n+(1-c)^{n} b_{c}(n)+2(n-1)(1-c) c^{n-1}+c-c^{n}-(n-1)(1-c) c^{n-1}+ \\
& \sum_{k=2}^{n-1}\left[(1-c) P_{k-1}^{n-1}+c P_{k}^{n-1}\right] b_{c}(k) \\
= & n+(1-c)^{n} b_{c}(n)+(n-1)(1-c) c^{n-1}+c-c^{n}+\sum_{k=2}^{n-1} P_{k}^{n} b_{c}(k) .
\end{aligned}
$$

Rewriting yields, for any $n \geq 2$,

$$
\left[1-(1-c)^{n}\right] b_{c}(n)=n+(n-1)(1-c) c^{n-1}+c-c^{n}+\sum_{k=2}^{n-1}\left(\begin{array}{l}
n \\
k
\end{array}\right)(1-c)^{k} c^{n-k} b_{c}(k) .
$$




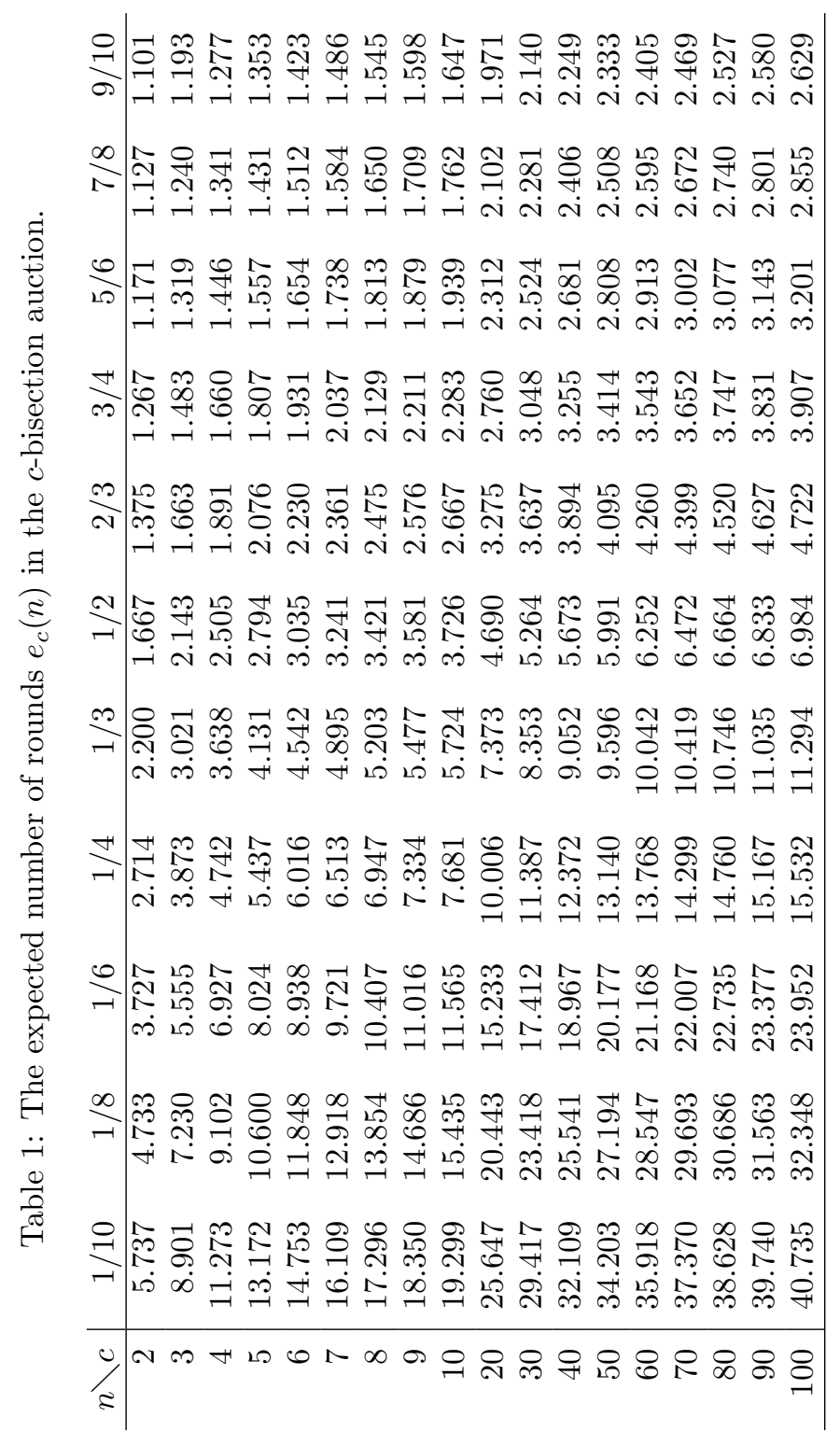




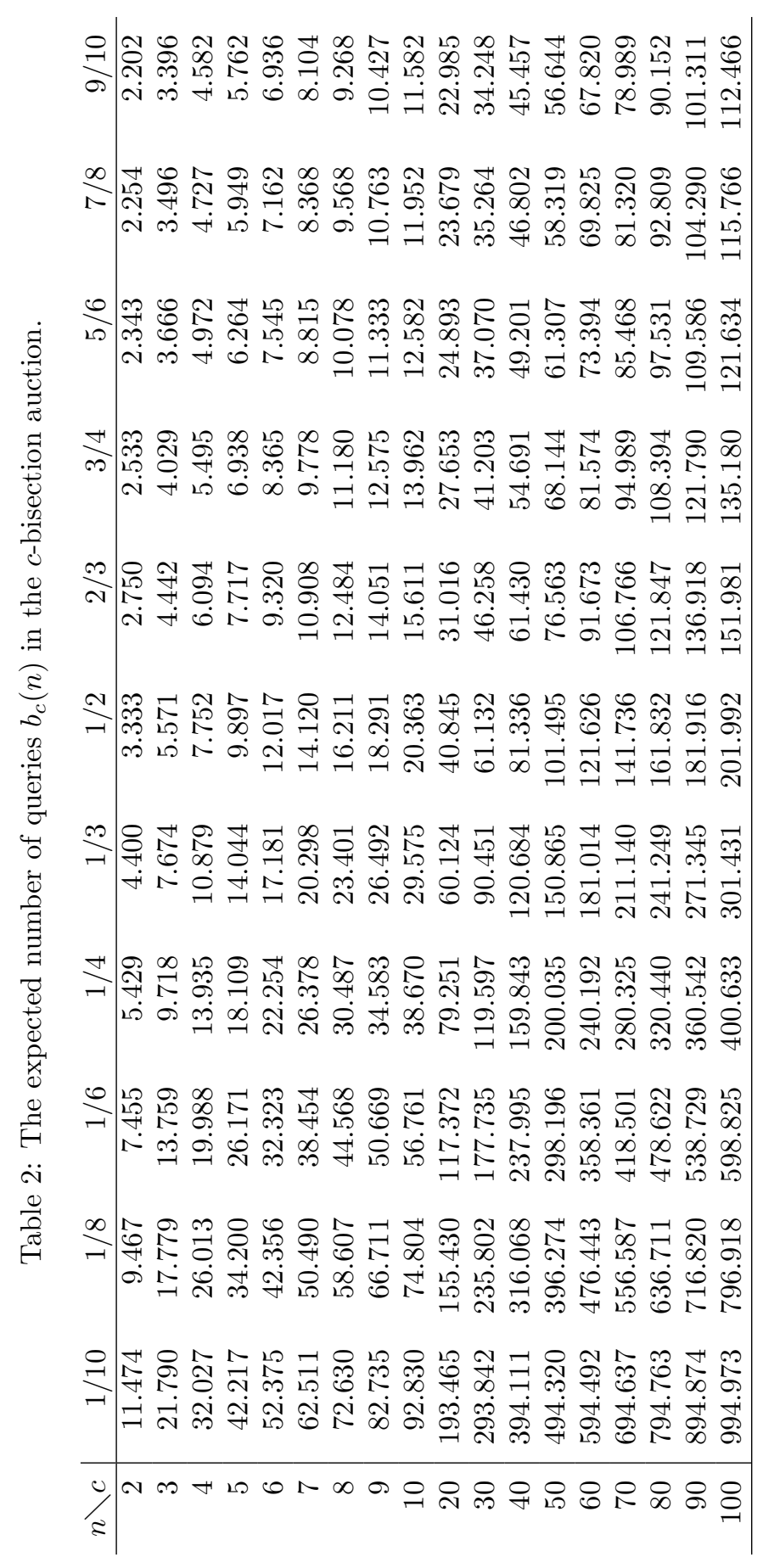

